\title{
A Case of Post-fundoplication Dysphagia: Another Possible Interpretation of the Manometric Findings
}

TO THE EDITOR: A recent case presentation, ${ }^{1}$ featuring a sample tracing from high-resolution manometry, reports findings for a 40-year-old male patient with persistent severe dysphagia and weight loss some 2 years after laparoscopic fundoplication. The author states the manometric findings supported a diagnosis of hypertensive lower esophageal sphincter (LES) with impaired esophagogastric junction (EGJ) relaxation. We would like to raise another possible interpretation of the findings: If the accompanying figure is typical of the entire manometric recording, then the pressure patternhas been incorrectly interpreted. In the image shown, the pressure pattern below the "high pressure zone" (at $44-47 \mathrm{~cm}$ ) is similar to the pressure pattern above the "high pressure zone," indicative of a curled or bent catheter. The irregular pressure pattern at the bottom of the image suggests the distal end of the catheter has not entered the stomach. This previously described "butterfly" pattern, ${ }^{2}$ with a mirror image of pressure at the level of catheter curling, needs to be identified during acquisition to enable adjustments to be made to avoid a technically inadequate study. Also, the observation that "a sudden increase was noted in the LES pressure up to $300 \mathrm{mmHg}$ after each wet swallow" is an artifact generated by an occluded sidehole of the bent catheter and is not attributable to the LES.

To correctlyevaluate the luminal EGJ pressures in this post fundoplication patient, a repeat manometryis advised, with gentle maneuvres to guide the catheter tip across the EGJ; if this fails, then catheter placement under endoscopic guidance is recommended. ${ }^{2}$ Additionally, a radiological contrast swallow will be helpful in identifying anyfunctional or anatomical anomaly, such as impaired esophageal outflow or a slipped fundoplication. ${ }^{4}$ Both objective assessments can better inform clinical management decisions.

This case presentation highlights the need for greater awareness of the technical pitfalls and limitations that can occur with high-resolution esophageal manometry data acquisition and interpretation. Pressure pattern recognition during the performance of studies and critical assessment of semi-automated data analysis output is required. In conclusion, the presented interesting case of post fundoplication dysphagia shows manometric data that should be interpreted with caution.

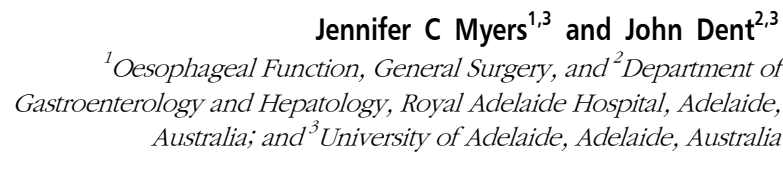

1. Jain M. An Interesting case of post-fundoplication dysphagia. J Neurogastroenterol Motil 2014;20:410-411.

2. Gyawali CP, Bredenoord AJ, Conklin JL, et al. Evaluation of esophageal motor function in clinical practice. Neurogastroenterol Motil 2013;25:99-133.

3. Bredenoord AJ, Hebbard GS. Technical aspects of clinical high-resolution manometry studies. Neurogastroenterol Motil 2012;24 (suppl 1):5-10.

4. Raeside MC, Madigan D, Myers JC, Devitt PG, Jamieson GG, Thompson SK. Post-fundoplication contrast studies: is there room for improvement? Br J Radiol 2012;85:792-799.

Conflicts of interest: None. 